Pacific

Journal of

Mathematics

\title{
APPROXIMATING THE MODULUS OF AN INNER FUNCTION
}

Geir Arne HJelle AND Artur Nicolau 


\title{
APPROXIMATING THE MODULUS OF AN INNER FUNCTION
}

\author{
Geir Arne HJelle AND ARtur Nicolau
}

\begin{abstract}
We show that the modulus of an inner function can be uniformly approximated in the unit disk by the modulus of an interpolating Blaschke product.
\end{abstract}

\section{Introduction}

Let $H^{\infty}$ be the algebra of bounded analytic functions in the unit disk $\mathbb{D}$. A function in $H^{\infty}$ is called inner if it has radial limit of modulus one at almost every point of the unit circle. A Blaschke product is an inner function of the form

$$
B(z)=z^{m} \prod_{n=1}^{\infty} \frac{\bar{z}_{n}}{\left|z_{n}\right|} \frac{z_{n}-z}{1-\bar{z}_{n} z},
$$

where $m$ is a nonnegative integer and $\left\{z_{n}\right\}$ is a sequence of points in $\mathbb{D} \backslash\{0\}$ satisfying the Blaschke condition $\sum_{n}\left(1-\left|z_{n}\right|\right)<\infty$. A classical result of O. Frostman tells that for any inner function $f$, there exists an exceptional set $E=E(f) \subset \mathbb{D}$ of logarithmic capacity zero such that the Möbius shift

$$
\frac{f-\alpha}{1-\bar{\alpha} f}
$$

is a Blaschke product for any $\alpha \in \mathbb{D} \backslash E$. See [Frostman 1935] or [Garnett 1981, p. 79]. Hence any inner function can be uniformly approximated by a Blaschke product.

A Blaschke product $B$ is called an interpolating Blaschke product if its zero set $\left\{z_{n}\right\}$ forms an interpolating sequence, that is, if for any bounded sequence of complex numbers $\left\{w_{n}\right\}$, there exists a function $f \in H^{\infty}$ such that $f\left(z_{n}\right)=w_{n}$, $n=1,2, \ldots$ A celebrated result by L. Carleson [1958] (or see [Garnett 1981, p. 287]) tells us that this holds precisely when two conditions are satisfied:

$$
\inf _{n \neq m}\left|\frac{z_{n}-z_{m}}{1-\bar{z}_{m} z_{n}}\right|>0,
$$

MSC2000: 30D50, 30E10.

Keywords: interpolating Blaschke product, Carleson contour, discretization, inner function, modulus.

Hjelle is partially supported by grants from the Research Council of Norway, projects \#155060 and \#166395. Nicolau is partially supported by MTM2005-00544 and 2005SGR00774. 
and there exists a constant $C$ such that $\sum_{z_{n} \in Q}\left(1-\left|z_{n}\right|\right)<C \ell(Q)$ for any Carleson square $Q$ of the form

$$
Q=\left\{r e^{i \theta}: 0<1-r<\ell(Q),\left|\theta-\theta_{0}\right|<\pi \ell(Q)\right\}
$$

where $\theta_{0} \in[0,2 \pi)$ and $0<\ell(Q)<1$. Although interpolating Blaschke products comprise a small subset of all Blaschke products, they play a central role in the theory of the algebra $H^{\infty}$. See the last three chapters of [Garnett 1981].

D. Marshall [1976] proved that any function $f \in H^{\infty}$ can be uniformly approximated by finite linear combinations of Blaschke products. That is, for any $\varepsilon>0$ there are constants $c_{1}, \ldots, c_{N}$ and Blaschke products $B_{1}, \ldots, B_{N}$ such that

$$
\left\|f-\sum_{i=1}^{N} c_{i} B_{i}\right\|_{\infty}<\varepsilon .
$$

Here the $\infty$-norm is given by $\|g\|_{\infty}=\sup \{|g(z)|: z \in \mathbb{D}\}$. This result was improved in [Garnett and Nicolau 1996] by showing that one can take each of $B_{1}, \ldots, B_{N}$ to be an interpolating Blaschke product. However the following problem remains open.

For any inner function $B$ and $\varepsilon>0$, is there an interpolating Blaschke product I such that $\|B-I\|_{\infty}<\varepsilon$ ?

This question was posed in [Garnett 1981, p. 430; Havin and Nikol'skiǔ 1994, pp. 268-269; Jones 1981; Nikol'skiǔ 1986, p. 202]. Here we provide a positive answer if one restricts attention to the modulus.

Theorem 1. Let $B$ be an inner function and $\varepsilon>0$. There exists an interpolating Blaschke product I such that

$$
|| B(z)|-| I(z)||<\varepsilon \quad \text { for all } z \in \mathbb{D} .
$$

The proof may be described as follows. The first step consists of constructing a system $\Gamma=\bigcup_{i} \Gamma_{i}$ of disjoint closed curves $\Gamma_{i} \subset \mathbb{D}$ such that arclength of $\Gamma$ is a Carleson measure, and verifying that

(a) $|B(z)|$ is uniformly small on hyperbolic disks of fixed radius centered at points of $\Gamma$, and

(b) in any hyperbolic disk of fixed radius centered at a point outside the union of the interiors of $\Gamma_{i}, \bigcup_{i}$ int $\Gamma_{i}$, there is a point $z$ where $|B(z)|$ is not small.

Write $B=B_{1} \cdot B_{2}$, where $B_{1}$ is the Blaschke product formed with the zeros of $B$ which are in $\bigcup_{i}$ int $\Gamma_{i}$. Statement (b) implies that $B_{2}$ is a finite product of interpolating Blaschke products. Since D. Marshall and A. Stray [1996] proved that any finite product of interpolating Blaschke products may be approximated by a single 
interpolating Blaschke product, the relevant zeros of $B$ lie in $\bigcup_{i}$ int $\Gamma_{i}$ : they are those of $B_{1}$. The construction of $\Gamma$ is a variation of the original corona construction introduced by L. Carleson [1962] (or see [Garnett 1981, pp. 342-347]).

Next, for each $i=1,2, \ldots$, let $\mu_{i}$ be the sum of harmonic measures in int $\Gamma_{i}$ from the zeros of $B_{1}$ contained in int $\Gamma_{i}$. Then the mass $\mu_{i}\left(\Gamma_{i}\right)$ is the total number of zeros of $B_{1}$ contained in int $\Gamma_{i}$. The second step consists of splitting $\Gamma_{i}$ as $\bigcup_{k} \Gamma_{i, k}$, where the pieces $\Gamma_{i, k}$ satisfy $\mu_{i}\left(\Gamma_{i, k}\right)=1, k=1,2, \ldots$, and choosing points $\xi_{i, k} \in \Gamma_{i, k}$ matching a certain moment of the measure $\mu_{i}$ on $\Gamma_{i, k}$. This choice may be compared with that of [Lyubarskii and Malinnikova 2001], where a related discretization argument is performed in a different context. Let $I_{1}$ be the Blaschke product with zeros $\xi_{i, k}, i, k=1,2, \ldots$ The last step of the proof is to use (b) above to show that $I_{1}$ is an interpolating Blaschke product and to use the location of $\left\{\xi_{i, k}\right\}$, as well as (a) above, to show that $\left|I_{1}(z) \cdot B_{2}(z)\right|$ approximates $|B(z)|$.

Besides the individual problem mentioned above, some questions concerning approximation by arguments of interpolating Blaschke products remain open. Let $B$ be an inner function.

A. Given $\varepsilon>0$, is there an interpolating Blaschke product I such that

$$
\|\operatorname{Arg} B-\operatorname{Arg} I\|_{\mathrm{BMO}(\partial \mathbb{D})}<\varepsilon ?
$$

B. Is there an interpolating Blaschke product I such that $\operatorname{Arg} B-\operatorname{Arg} I=\tilde{v}$, where $v \in L^{\infty}(\partial \mathbb{D})$ ?

C. Is there an interpolating Blaschke product I such that $\operatorname{Arg} B-\operatorname{Arg} I=u+\tilde{v}$, where $u, v \in L^{\infty}(\partial \mathbb{D})$ and $\|u\|_{\infty}<\pi / 2$ ?

A positive answer to Problem A would imply the main result of this note. Problem C was posed by in [Havin and Nikol'skir 1994; Nikol'skiur 1986] in connection with Toeplitz operators and complete interpolating sequences in model spaces. Problems B and C are discussed in the nice monograph by K. Seip [2004, p. 92].

\section{Construction of the contour}

The hyperbolic distance between two points $z, w \in \mathbb{D}$ is

$$
\beta(z, w)=\frac{1}{2} \log \frac{1+\rho(z, w)}{1-\rho(z, w)},
$$

where $\rho(z, w)$ is the pseudohyperbolic distance,

$$
\rho(z, w)=\left|\frac{z-w}{1-\bar{w} z}\right| .
$$


Recall that a positive measure $\mu$ in the unit disk is called a Carleson measure if there exists a constant $M=M(\mu)>0$ such that $\mu(Q) \leq M \ell(Q)$ for any Carleson square of the form (2). The infimum of the constants $M$ satisfying the inequality above is called the Carleson norm of the measure $\mu$ and it is denoted by $\|\mu\|_{C}$.

The main result of this section is a variant of the classical construction of the Carleson contour introduced by L. Carleson in his original proof of the corona theorem [1962] (or see [Garnett 1981, pp. 342-347]).

Lemma 2. Let $B \in H^{\infty}$ with $\|B\|_{\infty}=1$. Let $0<\varepsilon<1$ and $K>0$ be fixed constants. Then, there exist a constant $\delta=\delta(\varepsilon, K)>0$ and a system $\Gamma=\bigcup \Gamma_{i}$ of disjoint closed curves $\Gamma_{i}$ contained in $\mathbb{D}$ such that

(a) $|B(z)| \leq \varepsilon$ if $\inf _{i} \beta\left(z\right.$,int $\left.\Gamma_{i}\right) \leq K$;

(b) $\sup \{|B(w)|: \beta(w, z) \leq K+14\}>\delta$ if $z \notin \bigcup$ int $\Gamma_{i}$; and

(c) arclength $\mathrm{d} s_{\mid \Gamma}$ on $\Gamma$ is a Carleson measure with $\left\|\mathrm{d} s_{\mid \Gamma}\right\|_{C} \leq 68$.

Proof. The proof is essentially contained in [Nicolau and Suárez $\geq 2006$ ], but we sketch it for the convenience of the reader. Given a set $E \subset \mathbb{D}$, let $\Omega_{K}(E)$ denote the set of points that are at most at hyperbolic distance $K$ from the set $E$, that is,

$$
\Omega_{K}(E)=\left\{z: \inf _{w \in E} \beta(z, w) \leq K\right\} .
$$

Consider dyadic Carleson squares of the form

$$
Q_{n, j}=\left\{r e^{i \theta}: 1-2^{-n}<r<1,2 \pi j 2^{-n}<\theta<2 \pi(j+1) 2^{-n}\right\},
$$

for $j=0,1, \ldots, 2^{n}-1$ and $n=1,2, \ldots$, and their top halves

$$
T\left(Q_{n, j}\right)=\left\{r e^{i \theta} \in Q_{n, j}: r<1-2^{-n-1}\right\} .
$$

Let $0<\delta<\varepsilon$ be a constant to be fixed later. A dyadic Carleson square $Q$ will be called good if

$$
\sup \left\{|B(z)|: z \in \Omega_{K}(T(Q))\right\}>\varepsilon .
$$

The collection of good dyadic Carleson squares will be denoted by

$$
\left\{Q_{j}^{G}: j=1,2, \ldots\right\} .
$$

A dyadic Carleson square $Q$ will be called bad if

$$
\sup \left\{|B(z)|: z \in \Omega_{K}(T(Q))\right\}<\delta .
$$

We denote the collection of bad dyadic Carleson squares by $\left\{Q_{j}^{B}: j=1,2, \ldots\right\}$. The construction goes as follows. 

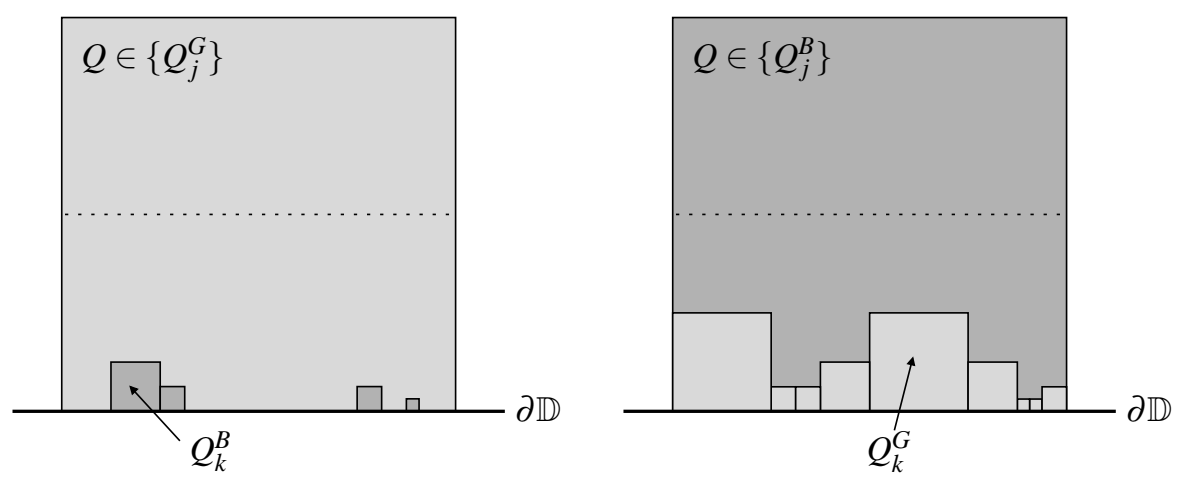

Figure 1. Choosing good and bad squares for constructing the contour.

Step 1. For each good dyadic Carleson square $Q=Q_{j}^{G}$, we choose the maximal bad dyadic Carleson squares $Q_{k}^{B}$ contained in $Q$. The main estimate needed is

$$
\sum_{Q_{k}^{B} \subset Q} \ell\left(Q_{k}^{B}\right) \leq \frac{1}{2} \ell(Q) .
$$

Since $|B(z)|<\delta$ if $z \in T\left(Q_{k}^{B}\right)$, while $|B(z)|>\varepsilon$ for some $z \in \Omega_{K}(T(Q))$, taking $\delta=\delta(\varepsilon, K)$ sufficiently small, standard arguments lead to (3). See [Nicolau and Suárez $\geq 2006$, Lemma 2.1] for details.

Step 2. For each bad dyadic Carleson square $Q=Q_{j}^{B}$, we choose the maximal good dyadic Carleson squares $Q_{k}^{G}$ contained in $Q$. This family is denoted by $G(Q)=\left\{Q_{k}^{G}: k=1,2, \ldots\right\}$.

So, from each good dyadic Carleson square we move to bad ones fulfilling the estimate (3) and from each bad one we again move to good ones. See Figure 1. Now for each bad square $Q=Q_{j}^{B}$, let

$$
R(Q)=Q \backslash \overline{\bigcup_{G(Q)} Q_{k}^{G}} \quad \text { and } \quad R=\bigcup_{j} R\left(Q_{j}^{B}\right) .
$$

Finally, decompose $R$ into its connected components $R_{i}$ and denote $\Gamma_{i}=\partial R_{i}$, $i=1,2, \ldots$. Observe that each $\Gamma_{i}$ consists of pieces of boundaries of dyadic Carleson squares. See Figure 2. By construction if $z \in R$ we have

$$
\sup \{|B(w)|: \beta(w, z) \leq K\} \leq \varepsilon
$$

and hence part (a) in the statement follows. Similarly, if $z \notin R$, the point $z$ is not in the top part of a bad dyadic Carleson square. As the hyperbolic diameter of a top part of a Carleson square is uniformly bounded, say by 14 , we deduce that there exists $w \in \mathbb{D}$ with $\beta(z, w) \leq K+14$ such that $|B(w)|>\delta$. Hence statement (b) 


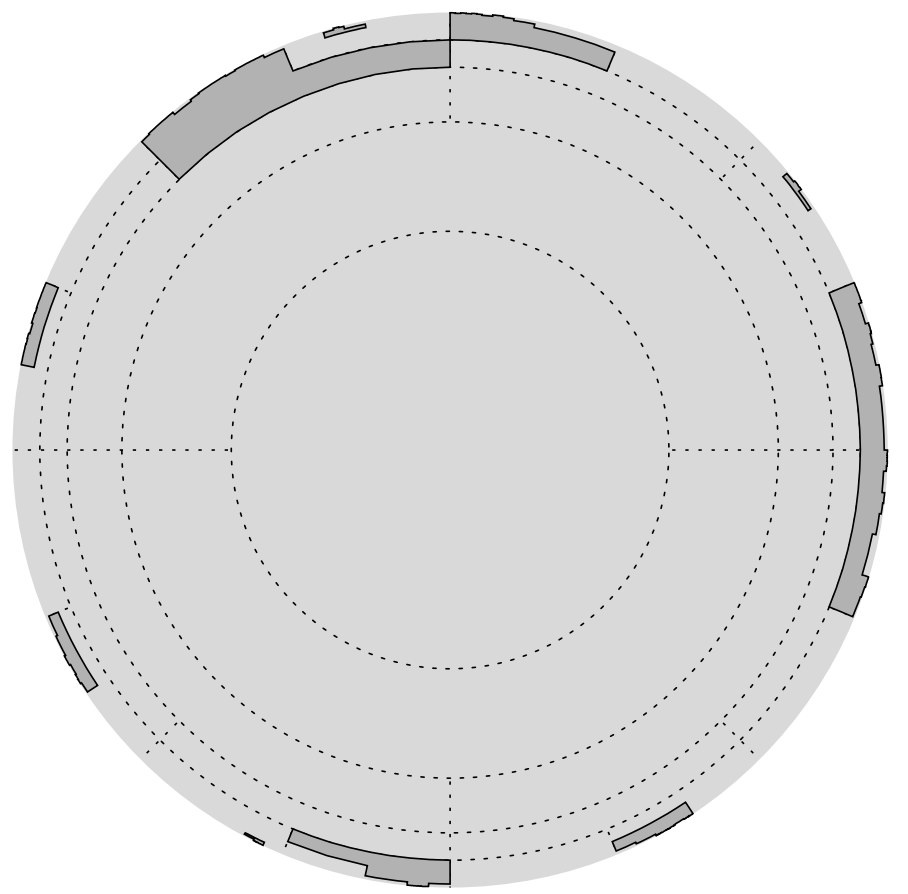

Figure 2. The unit disk, some dyadic Carleson contours and an example of a contour.

follows. Since the length of $\partial R(Q)$ is bounded by $17 \ell(Q)$, the scaling (3) shows that for any bad dyadic square $Q$, one has

$$
\sum_{Q_{j}^{B} \subsetneq Q}\left|\partial R\left(Q_{j}^{B}\right)\right| \leq 17 \ell(Q) .
$$

Then easy geometric considerations show that arclength on $\bigcup \Gamma_{i}$ is a Carleson measure and its Carleson norm is smaller than 68 .

\section{Construction of the interpolating Blaschke product}

We now use Lemma 2 to construct a contour $\Gamma$. Note that by Frostman's Theorem we can assume that $B$ is a Blaschke product. Given $\varepsilon>0$, let $N$ be a big constant dependent on $\varepsilon$ to be fixed later. Apply Lemma 2 with $\varepsilon / 2$ and $2 N$ instead of $\varepsilon$ and $K$ to obtain $\Gamma$ and $\delta>0$ such that

(a) $|B(z)|<\varepsilon / 2$ if $\beta(z$, int $\Gamma) \leq 2 N$,

(b) $\sup \{|B(w)|: \beta(w, z) \leq 2 N+14\}>\delta$ if $z \notin$ int $\Gamma$, and

(c) arclength on $\Gamma$ is a Carleson measure with Carleson norm $\left\|\mathrm{d} s_{\mid \Gamma}\right\|_{C} \leq 68$. 
With the contour $\Gamma$ in place, we want to construct the interpolating Blaschke product $I$. Split $B$ into two Blaschke products $B_{1}$ and $B_{2}$. That is $B=B_{1} \cdot B_{2}$, where $B_{1}$ is formed with the zeros $\left\{z_{n}\right\}$ of $B$ that lie inside int $\Gamma$ and at hyperbolic distance more than 1 from the contour $\Gamma$. For each zero $z$ of $B_{2}$, part (b) provides a point $w \in \mathbb{D}, \beta(w, z) \leq 2 N+15$ such that $\left|B_{2}(w)\right| \geq|B(w)|>\delta$. This implies that $B_{2}$ is a finite product of interpolating Blaschke products; [Mortini and Nicolau 2004, Theorem 2.2].

Hence the dangerous part of $B$ will be $B_{1}$, which has all its zeros contained deeply inside the contour $\Gamma$. We want to mimic the behavior of $\left|B_{1}\right|$ by constructing a Blaschke product $I_{1}$ with zeros on $\Gamma$. To this end, for each component $\Gamma_{i}$ of the contour we consider the measure

$$
\mathrm{d} \mu_{i}(\xi)=\sum_{\substack{z_{n} \in \operatorname{int} \Gamma_{i} \\ \beta\left(z_{n}, \Gamma_{i}\right)>1}} \omega\left(z_{n}, \xi ; \text { int } \Gamma_{i}\right)
$$

defined for $\xi \in \Gamma_{i}$. Here $\omega(z, \xi ; \Omega)$ denotes the harmonic measure from the point $z \in \Omega$ in the domain $\Omega \subseteq \mathbb{D}$. Clearly $\mu_{i}\left(\Gamma_{i}\right)$ will be equal to the number of zeros $z_{n}$ of $B_{1}$ inside $\Gamma_{i}$. Next we split $\Gamma_{i}$ into disjoint $\operatorname{arcs} \Gamma_{i, k}$ such that $\mu_{i}\left(\Gamma_{i, k}\right)=1$ for each $k$. This is illustrated in Figure 3. On each such arc we locate one zero $\xi_{i, k}$ of $I_{1}$ such that

$$
1-\left|\xi_{i, k}\right|^{2}=\int_{\Gamma_{i, k}}\left(1-|\xi|^{2}\right) \mathrm{d} \mu_{i}(\xi)
$$

This will in general not determine the points $\xi_{i, k}$ uniquely. However, there seems to be a lot of freedom for placing the zeros of $I_{1}$ in this construction, and the condition (4) will be sufficient for our purposes.

Let $I_{1}$ be the Blaschke product with the zeros $\xi_{i, k}$, and factor $I_{1}=I_{1}^{o} \cdot I_{1}^{e}$ where $I_{1}^{o}$ is the Blaschke product with zeros $\xi_{i, k}$ with $k$ odd, while $I_{1}^{e}$ is the Blaschke product with zeros $\xi_{i, k}$ with $k$ even. In Figure $3, I_{1}^{o}$ has its zeros placed in the dark arcs, while the zeros of $I_{1}^{e}$ are placed in the light arcs. We claim that both $I_{1}^{o}$ and $I_{1}^{e}$ are interpolating Blaschke products, and hence $I_{1}$ can be approximated by an interpolating Blaschke product [Marshall and Stray 1996]. To show this claim we will observe that their zero sets satisfy the two conditions of Carleson's theorem [1958] stated in the introduction.

In this case, the existence of a constant $C$ as in Carleson's criterion (see top of page 104) follows from the fact that arclength is a Carleson measure on $\Gamma$, while inequality (1) follows from the following lemma and the geometry of the contour.

Lemma 3. The hyperbolic length, $\ell_{\beta}\left(\Gamma_{i, k}\right)$, of $\Gamma_{i, k}$ is bounded from below:

$$
\ell_{\beta}\left(\Gamma_{i, k}\right) \geq \delta^{2 \exp (2(2 N+14))} .
$$




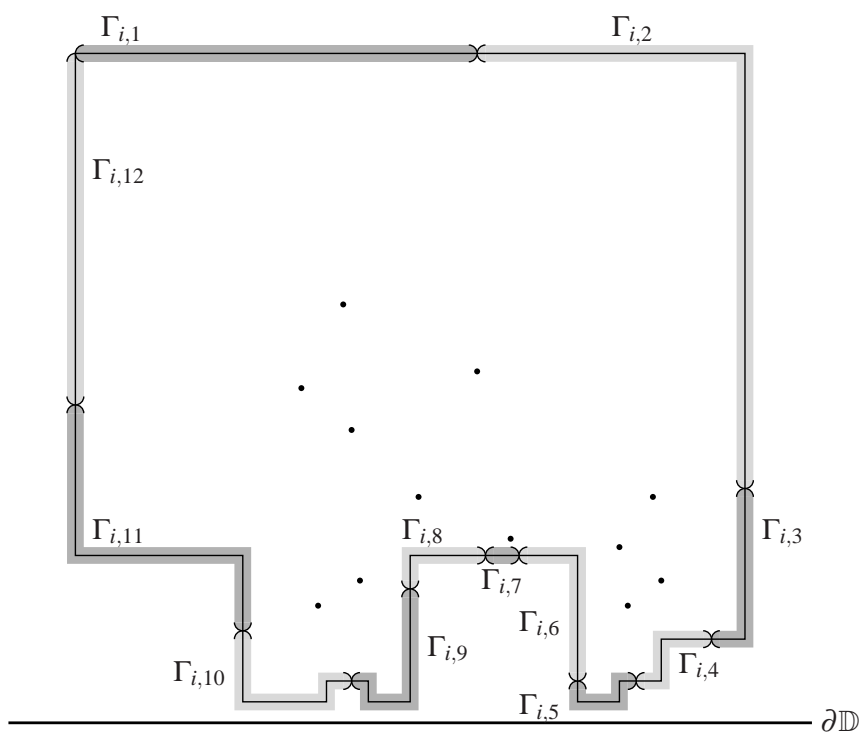

Figure 3. Each component $\Gamma_{i}$ of the contour is split into arcs $\Gamma_{i, k}$ such that the $\mu$-measure of each arc is 1 .

Proof. We first show that for any point $w \in \Gamma,\left|B_{1}(w)\right|$ is bounded from below by some constant depending only on $\delta$ and $N$. To see this, recall that there is a point $\zeta$ such that $\beta(\zeta, w) \leq 2 N+14$ and $\left|B_{1}(\zeta)\right| \geq|B(\zeta)|>\delta$. Consider

$$
\log \left|B_{1}(w)\right|^{-1}=\sum \log \rho\left(w, z_{n}\right)^{-1},
$$

where the sum is taken over all zeros $z_{n}$ of $B_{1}$. As $w$ is separated from the zeros of $B_{1}$,

$$
\log \rho\left(w, z_{n}\right)^{-1} \leq 1-\rho\left(w, z_{n}\right)^{2}
$$

Furthermore,

$$
\rho\left(w, z_{n}\right) \geq \frac{\rho\left(z_{n}, \zeta\right)-\rho(\zeta, w)}{1-\rho\left(z_{n}, \zeta\right) \rho(\zeta, w)} \geq \frac{\rho\left(z_{n}, \zeta\right)-C}{1-C \rho\left(z_{n}, \zeta\right)},
$$

where $C=\frac{e^{2(2 N+14)}-1}{e^{2(2 N+14)}+1}<1$. Hence

$$
\begin{aligned}
\log \rho\left(w, z_{n}\right)^{-1} & \leq \frac{\left(1-\rho\left(z_{n}, \zeta\right)^{2}\right)\left(1-C^{2}\right)}{\left(1-C \rho\left(z_{n}, \zeta\right)\right)^{2}} \leq \frac{1+C}{1-C}\left(1-\rho\left(z_{n}, \zeta\right)^{2}\right) \\
& \leq 2 \frac{1+C}{1-C} \log \rho\left(z_{n}, \zeta\right)^{-1}=2 e^{2(2 N+14)} \log \rho\left(z_{n}, \zeta\right)^{-1}
\end{aligned}
$$

and we see that $\left|B_{1}(w)\right| \geq \delta^{2 \exp (2(2 N+14))}$. 
Intuitively, this lower bound for the values of $\left|B_{1}\right|$ should imply that the $\operatorname{arcs} \Gamma_{i, k}$ cannot be too short hyperbolically. To make this observation rigorous we argue as follows. Using that the harmonic measure $\omega$ is positive and harmonic, we have that for any $z \in$ int $\Gamma_{i}$,

$$
\omega\left(z, \Gamma_{i, k} ; \text { int } \Gamma_{i}\right) \leq \omega\left(z, \Gamma_{i, k} ; \mathbb{D} \backslash \Gamma_{i, k}\right) \leq \frac{\int_{\Gamma_{i, k}} \log \left|\frac{z-w}{1-\bar{w} z}\right|^{-1} \frac{|\mathrm{d} w|}{1-|w|^{2}}}{\min _{z \in \Gamma_{i, k}} \int_{\Gamma_{i, k}} \log \left|\frac{z-w}{1-\bar{w} z}\right|^{-1} \frac{|\mathrm{d} w|}{1-|w|^{2}}}
$$

and

where

$$
\begin{aligned}
1=\mu_{i}\left(\Gamma_{i, k}\right) & =\sum_{z_{n} \in \operatorname{int} \Gamma_{i}} \omega\left(z_{n}, \Gamma_{i, k} ; \operatorname{int} \Gamma_{i}\right) \\
& \leq \frac{1}{C_{i, k}} \int_{\Gamma_{i, k}} \log \left(\prod_{z_{n} \in \operatorname{int} \Gamma_{i}}\left|\frac{z_{n}-w}{1-\bar{w} z_{n}}\right|^{-1}\right) \frac{|\mathrm{d} w|}{1-|w|^{2}},
\end{aligned}
$$

$$
C_{i, k}=\min _{z \in \Gamma_{i, k}} \int_{\Gamma_{i, k}} \log \left|\frac{z-w}{1-\bar{w} z}\right|^{-1} \frac{|\mathrm{d} w|}{1-|w|^{2}}
$$

is a constant dependent on $\Gamma_{i, k}$. Let $B_{1, i}$ denote the Blaschke product with the zeros of $B_{1}$ that fall inside the component $\Gamma_{i}$. Then, for $w \in \Gamma_{i}$,

$\log \left(\prod_{z_{n} \in \operatorname{int} \Gamma_{i}}\left|\frac{z_{n}-w}{1-\bar{w} z_{n}}\right|^{-1}\right)=\log \left|B_{1, i}(w)\right|^{-1} \leq \log \left|B_{1}(w)\right|^{-1} \leq 2 e^{2(2 N+14)} \log \delta^{-1}$.

Thus

$$
1 \leq \frac{1}{C_{i, k}} 2 e^{2(2 N+14)} \log \delta^{-1} \int_{\Gamma_{i, k}} \frac{|\mathrm{d} w|}{1-|w|^{2}}=\frac{1}{C_{i, k}} 2 e^{2(2 N+14)} \log \delta^{-1} \ell_{\beta}\left(\Gamma_{i, k}\right)
$$

such that

$$
\ell_{\beta}\left(\Gamma_{i, k}\right) \geq \frac{C_{i, k}}{2 e^{2(2 N+14)} \log \delta^{-1}} .
$$

To estimate $C_{i, k}$ we use the substitution $\xi=\varphi_{z}(w)=(z-w) /(1-\bar{w} z)$ and the conformal invariance of the hyperbolic metric. A calculation then gives that

$$
C_{i, k} \geq \log \left(\tanh \ell_{\beta}\left(\Gamma_{i, k}\right)\right) \ell_{\beta}\left(\Gamma_{i, k}\right),
$$

which implies the desired bound, $\ell_{\beta}\left(\Gamma_{i, k}\right) \geq \delta^{2 \exp (2(2 N+14))}$.

\section{Proof of the approximation}

In this section we will show that the constructed function, $I=I_{1} \cdot B_{2}$, approximates the given Blaschke product uniformly in modulus. We first claim that it suffices to prove Theorem 1 for points $z \in \mathbb{D}$ far away from the contour. Indeed, assume that we can prove that

$$
|| B_{1}(z)|-| I_{1}(z)||<\varepsilon / 2
$$


for all $z$ such that $\beta(z$, int $\Gamma) \geq 2 N$, where $N$ is as in the construction of the contour. Then, for points $z$ with $\beta(z$, int $\Gamma)=2 N$,

$$
\begin{aligned}
|I(z)| & =\left(\left|I_{1}(z)\right|-\left|B_{1}(z)\right|+\left|B_{1}(z)\right|\right)\left|B_{2}(z)\right| \\
& \leq|| B_{1}(z)|-| I_{1}(z)||+|B(z)|<\varepsilon / 2+\varepsilon / 2=\varepsilon .
\end{aligned}
$$

By the maximum principle $|I(z)|<\varepsilon$ for all $z \in \Omega_{2 N}($ int $\Gamma$ ) as well. Hence

$$
|| B(z)|-| I(z)||=|| B_{1}(z)|-| I_{1}(z)||\left|B_{2}(z)\right|< \begin{cases}\varepsilon / 2 & \text { if } \beta(z, \text { int } \Gamma) \geq 2 N, \\ \varepsilon & \text { if } \beta(z, \text { int } \Gamma)<2 N .\end{cases}
$$

So Theorem 1 follows from (5).

The rest of the paper will be dedicated to prove that (5) holds. Fix a point $z$ such that $\beta(z$, int $\Gamma) \geq 2 N$. We will consider the logarithm of $\left|B_{1}\right|$. Since all the zeros of $B_{1}$ lie inside the contour $\Gamma, \log \left|\left(z-z_{n}\right) /\left(1-\bar{z}_{n} z\right)\right|$ is harmonic inside $\Gamma$ as a function of $z_{n}$. Hence

$$
\log \left|B_{1}(z)\right|=\sum_{j} \log \left|\frac{z-z_{n}}{1-\bar{z}_{n} z}\right|=\int_{\Gamma} \log \left|\frac{z-\xi}{1-\bar{\xi} z}\right| \mathrm{d} \mu(\xi),
$$

where $\mathrm{d} \mu=\sum_{i} \mathrm{~d} \mu_{i}$. As the $\mu$-measure of each $\operatorname{arc} \Gamma_{i, k}$ is 1 , we have

$$
\text { (6) } \begin{aligned}
\log \left|B_{1}(z)\right|-\log \left|I_{1}(z)\right| & =\int_{\Gamma} \log \left|\frac{z-\xi}{1-\bar{\xi} z}\right| \mathrm{d} \mu(\xi)-\sum_{i, k} \log \left|\frac{z-\xi_{i, k}}{1-\bar{\xi}_{i, k}}\right| \\
& =\sum_{i, k} \int_{\Gamma_{i, k}}\left(\log \left|\frac{z-\xi}{1-\bar{\xi}_{z}}\right|-\log \left|\frac{z-\xi_{i, k}}{1-\bar{\xi}_{i, k} z}\right|\right) \mathrm{d} \mu(\xi) \\
& =\sum_{i, k} \int_{\Gamma_{i, k}} \log \frac{\rho(z, \xi)}{\rho\left(z, \xi_{i, k}\right)} \mathrm{d} \mu(\xi) \stackrel{\text { def }}{=} \sum_{i, k} H_{i, k}(z) .
\end{aligned}
$$

To estimate this sum we consider different types of arcs. By $Q_{z}$ we denote the Carleson square with $z$ as the midpoint on the top-side. We say that an $\operatorname{arc} \Gamma_{i, k}$ is in the class $\mathscr{B}$ if $\Gamma_{i, k} \subset 2^{N} Q_{z}$. Note that since $\beta(z$, int $\Gamma) \geq 2 N$, this implies that such an arc lies very close to the boundary. The rest of the arcs we split into short and long arcs. For $n \geq N+1$ define

$$
\begin{aligned}
& \mathscr{S}_{n}=\left\{\Gamma_{i, k}: \ell_{\beta}\left(\Gamma_{i, k}\right)<1, \Gamma_{i, k} \subset 2^{n} Q_{z}\right\} \backslash\left(\mathscr{B} \cup \bigcup_{i<n} \mathscr{Y}_{i}\right), \\
& \mathscr{L}_{n}=\left\{\Gamma_{i, k}: \ell_{\beta}\left(\Gamma_{i, k}\right) \geq 1, \Gamma_{i, k} \subset 2^{n} Q_{z}\right\} \backslash\left(\mathscr{B} \cup \bigcup_{i<n} \mathscr{L}_{i}\right) .
\end{aligned}
$$

Consult Figure 4 for some examples of this classification. This partition is such that each arc $\Gamma_{i, k}$ belongs to one and only one of the classes $\mathscr{B}, \mathscr{S}_{n}$ and $\mathscr{L}_{n}$, with 


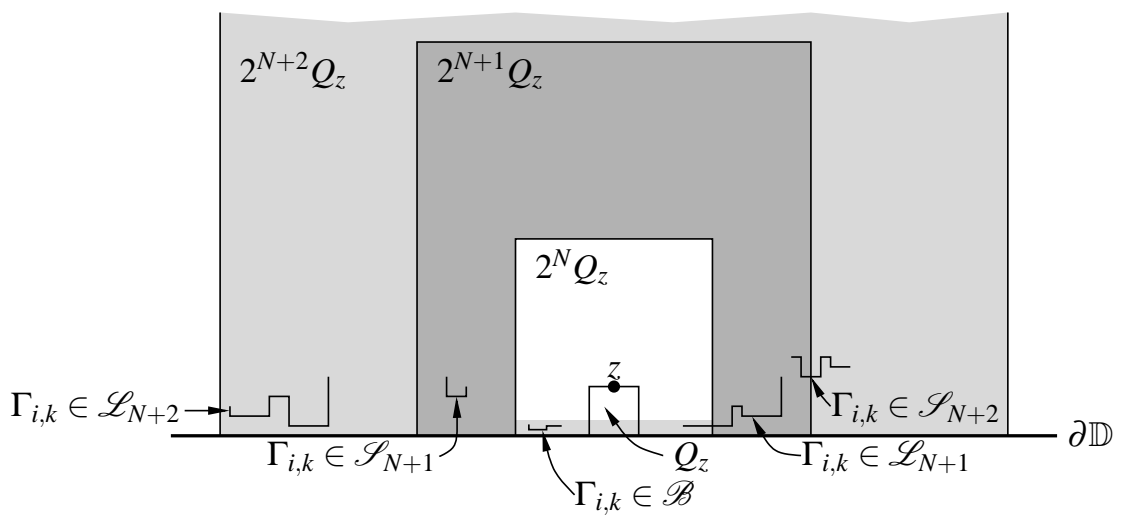

Figure 4. We divide the $\operatorname{arcs} \Gamma_{i, k}$ into classes denoted $\mathscr{B}, \mathscr{Y}_{n}$ and $\mathscr{L}_{n}$.

$n \geq N+1$. Hence we may decompose the sum (6) as

$$
\sum_{i, k} H_{i, k}(z)=\sum_{\Gamma_{i, k} \in \mathscr{\Re}} H_{i, k}(z)+\sum_{n=N+1}^{\infty}\left(\sum_{\Gamma_{i, k} \in \mathscr{S}_{n}} H_{i, k}(z)+\sum_{\Gamma_{i, k} \in \mathscr{L}_{n}} H_{i, k}(z)\right) .
$$

Our goal is to show that the absolute value of the left hand side is small. To accomplish this we will show that each of the terms

$$
\left|\sum_{\Gamma_{i, k} \in \mathscr{B}} H_{i, k}(z)\right|, \quad\left|\sum_{n=N+1}^{\infty} \sum_{\Gamma_{i, k} \in \mathscr{Y}_{n}} H_{i, k}(z)\right| \text { and }\left|\sum_{n=N+1}^{\infty} \sum_{\Gamma_{i, k} \in \mathscr{L}_{n}} H_{i, k}(z)\right|
$$

are small.

We begin with the boundary $\operatorname{arcs} \Gamma_{i, k} \in \mathscr{B}$. Using that $\log (1-t)=-t+\mathscr{O}\left(t^{2}\right)$ we get

$$
\begin{aligned}
\sum_{\Gamma_{i, k} \in \mathscr{P}} \int_{\Gamma_{i, k}} \log & \frac{\rho(z, \xi)}{\rho\left(z, \xi_{i, k}\right)} \mathrm{d} \mu(\xi) \\
& =-\frac{1}{2} \sum_{\Gamma_{i, k} \in \mathscr{B}} \int_{\Gamma_{i, k}}\left(1-\frac{\rho(z, \xi)^{2}}{\rho\left(z, \xi_{i, k}\right)^{2}}+\mathcal{O}\left(\left(1-\frac{\rho(z, \xi)^{2}}{\rho\left(z, \xi_{i, k}\right)^{2}}\right)^{2}\right)\right) \mathrm{d} \mu(\xi)
\end{aligned}
$$

Taking absolute values,

$$
\begin{aligned}
\left|\sum_{\Gamma_{i, k} \in \mathscr{B}} \int_{\Gamma_{i, k}} \log \frac{\rho(z, \xi)}{\rho\left(z, \xi_{i, k}\right)} \mathrm{d} \mu(\xi)\right| \leq \frac{1}{2}\left|\sum_{\Gamma_{i, k} \in \mathscr{B}} \int_{\Gamma_{i, k}} 1-\frac{\rho(z, \xi)^{2}}{\rho\left(z, \xi_{i, k}\right)^{2}} \mathrm{~d} \mu(\xi)\right| \\
+\frac{1}{2}\left|\sum_{\Gamma_{i, k} \in \mathscr{乃}} \int_{\Gamma_{i, k}} \mathrm{O}\left(\left(1-\frac{\rho(z, \xi)^{2}}{\rho\left(z, \xi_{i, k}\right)^{2}}\right)^{2}\right) \mathrm{d} \mu(\xi)\right| \stackrel{\text { def }}{=} E_{\Re_{B}, 1}+E_{\mathscr{B}, 2},
\end{aligned}
$$


where we define $E_{\mathscr{B}, 1}$ and $E_{\mathscr{乃}, 2}$ for convenience. At first we focus on the term $E_{\mathscr{B}, 1}$. Note that since $z$ is far away from $\xi_{i, k} \in \Gamma_{i}$, the expression $\rho\left(z, \xi_{i, k}\right)^{-2}$ is bounded above, say by 2 . By expanding $1-\rho(z, \xi)^{2}$ and $1-\rho\left(z, \xi_{i, k}\right)^{2}$, we can write

$$
\begin{aligned}
E_{\mathscr{乃}, 1} & \leq \sum_{\Gamma_{i, k} \in \mathscr{P}}\left|\int_{\Gamma_{i, k}}\left(1-|z|^{2}\right)\left(\frac{1-|\xi|^{2}}{|1-\bar{\xi} z|^{2}}-\frac{1-\left|\xi_{i, k}\right|^{2}}{\left|1-\bar{\xi}_{i, k} z\right|^{2}}\right) \mathrm{d} \mu(\xi)\right| \\
& =\sum_{\Gamma_{i, k} \in \mathscr{P}}\left|\int_{\Gamma_{i, k}}\left(1-|z|^{2}\right)\left(\frac{1-|\xi|^{2}}{|1-\bar{\xi} z|^{2}}-\frac{1-|\xi|^{2}}{\left|1-\bar{\xi}_{i, k} z\right|^{2}}+\frac{\left|\xi_{i, k}\right|^{2}-|\xi|^{2}}{\left|1-\bar{\xi}_{i, k} z\right|^{2}}\right) \mathrm{d} \mu(\xi)\right|
\end{aligned}
$$

By the placement, (4), of the zeros $\xi_{i, k}$, the integral of the last term is zero. We now move the modulus under the integral to get

$$
E_{\mathscr{B}, 1} \leq\left(1-|z|^{2}\right) \sum_{\Gamma_{i, k} \in \mathscr{乃}} \int_{\Gamma_{i, k}}\left(1-|\xi|^{2}\right)\left|\frac{1}{|1-\bar{\xi} z|^{2}}-\frac{1}{\left|1-\bar{\xi}_{i, k} z\right|^{2}}\right| \mathrm{d} \mu(\xi) .
$$

Because $\xi$ and $\xi_{i, k}$ should be close to each other in some sense, compared to $z$, we suspect some cancellation. Therefore we use the estimate

$$
\left|\frac{1}{\left|1-\bar{\xi}_{z}\right|^{2}}-\frac{1}{\left|1-\bar{\xi}_{i, k} z\right|^{2}}\right| \leq \frac{2\left|\xi-\xi_{i, k}\right|}{(1-|z|)^{3}}
$$

and the more trivial inequalities $\left|\xi-\xi_{i, k}\right| \leq \ell\left(\Gamma_{i, k}\right)$ and $1-|z|^{2} \leq 2(1-|z|)$ to obtain

$$
E_{\Re, 1} \leq 2^{3}(1-|z|)^{-2} \sum_{\Gamma_{i, k} \in \mathscr{B}} \ell\left(\Gamma_{i, k}\right) \int_{\Gamma_{i, k}}(1-|\xi|) \mathrm{d} \mu(\xi) .
$$

All the arcs $\Gamma_{i, k} \in \mathscr{B}$ are contained in a rectangle at the boundary with height $2^{-2 N}(1-|z|)$ and width $2^{N}(1-|z|)$. Using that $1-|\xi| \leq 2^{-2 N}(1-|z|)$ and that the arclength $\mathrm{d} s_{\mid \Gamma}$ is a Carleson measure, we then get

$$
E_{\Re, 1} \leq 2^{3}\left\|\mathrm{~d} s_{\mid \Gamma}\right\|_{C} \cdot 2^{-N}
$$

where $\left\|\mathrm{d} s_{\mid \Gamma}\right\|_{C}$ is the Carleson norm of arclength on $\Gamma$.

Next we focus our attention on the higher-order terms, and give the estimate for $E_{\mathscr{B}, 2}$. From (7) and (8) and the inequality $(a+b)^{2} \leq 2\left(a^{2}+b^{2}\right)$ we see that $E_{\mathscr{B}, 2}$ is bounded by a fixed multiple of

$$
\begin{aligned}
\left(1-|z|^{2}\right)^{2} \sum_{\Gamma_{i, k} \in \mathscr{P}} \int_{\Gamma_{i, k}}\left(1-|\xi|^{2}\right)^{2} \mid & \frac{1}{\left|1-\bar{\xi}_{z}\right|^{2}}-\left.\frac{1}{\left|1-\bar{\xi}_{i, k} z\right|^{2}}\right|^{2} \mathrm{~d} \mu(\xi) \\
& +\left(1-|z|^{2}\right)^{2} \sum_{\Gamma_{i, k} \in \mathscr{P}} \int_{\Gamma_{i, k}} \frac{\left(\left|\xi_{i, k}\right|^{2}-|\xi|^{2}\right)^{2}}{\left|1-\bar{\xi}_{i, k} z\right|^{4}} \mathrm{~d} \mu(\xi) .
\end{aligned}
$$


For the first term, we use as above the estimate (10) as well as $1-|\xi| \leq 2^{-2 N}(1-|z|)$ and $\left|\xi-\xi_{i, k}\right| \leq 2 \cdot 2^{N}(1-|z|)$. Then we find

$$
\begin{aligned}
& \left(1-|z|^{2}\right)^{2} \sum_{\Gamma_{i, k} \in \mathscr{P}} \int_{\Gamma_{i, k}}\left(1-|\xi|^{2}\right)^{2}\left|\frac{1}{|1-\bar{\xi} z|^{2}}-\frac{1}{\left|1-\bar{\xi}_{i, k} z\right|^{2}}\right|^{2} \mathrm{~d} \mu(\xi) \\
& \quad \leq 2^{4} \cdot 2^{-N} \cdot\left(1-|z|^{2}\right) \sum_{\Gamma_{i, k} \in \mathscr{乃}} \int_{\Gamma_{i, k}}\left(1-|\xi|^{2}\right)\left|\frac{1}{|1-\bar{\xi} z|^{2}}-\frac{1}{\left|1-\bar{\xi}_{i, k} z\right|^{2}}\right| \mathrm{d} \mu(\xi) .
\end{aligned}
$$

The last sum is just (9), and by the earlier argument the last expression is bounded by $2^{7}\left\|\mathrm{~d} s_{\mid \Gamma}\right\|_{C} \cdot 2^{-2 N}$.

For the second term we use that $\left|1-\bar{\xi}_{i, k} z\right| \geq 1-|z|,|| \xi_{i, k}|-| \xi|| \leq 2^{-2 N}(1-|z|)$ and ||$\xi_{i, k}|-| \xi|| \leq \ell\left(\Gamma_{i, k}\right)$ to arrive at

$$
\begin{array}{rl}
\left(1-|z|^{2}\right)^{2} \sum_{\Gamma_{i, k} \in \mathscr{P}} \int_{\Gamma_{i, k}} \frac{\left(\left|\xi_{i, k}\right|^{2}-|\xi|^{2}\right)^{2}}{\mid 1}-\left.\bar{\xi}_{i, k} z\right|^{4} & \mathrm{~d} \mu(\xi) \\
& \leq\left. 2^{4}(1-|z|)^{-2} \sum_{\Gamma_{i, k} \in \mathscr{P}} \int_{\Gamma_{i, k}}|| \xi_{i, k}|-| \xi\right|^{2} \mathrm{~d} \mu(\xi) \\
& \leq 2^{4} \cdot 2^{-2 N}(1-|z|)^{-1} \sum_{\Gamma_{i, k} \in \mathscr{P}} \ell\left(\Gamma_{i, k}\right) \leq 2^{4}\left\|\mathrm{~d} s_{\mid \Gamma}\right\|_{C} \cdot 2^{-N}
\end{array}
$$

Thus we get $E_{\dddot{乃}, 2} \leq C\left(2^{4}+1\right)\left\|\mathrm{d} s_{\mid \Gamma}\right\|_{C} \cdot 2^{-N}$ for big $N$.

For the short arcs $\Gamma_{i, k} \in \mathscr{Y}_{n}, n \geq N+1$, we will use similar estimates as above, but we do not need to be as delicate. For these arcs we can use the inequality $|\log x| \leq\left|1-x^{2}\right|$, which holds for $x$ far away from zero, to obtain

$$
\begin{aligned}
E_{\varphi} \stackrel{\text { def }}{=}\left|\sum_{n=N+1}^{\infty} \sum_{\Gamma_{i, k} \in \mathscr{S}_{n}} \int_{\Gamma_{i, k}} \log \frac{\rho(z, \xi)}{\rho\left(z, \xi_{i, k}\right)} \mathrm{d} \mu(\xi)\right| \\
\leq \sum_{n=N+1}^{\infty} \sum_{\Gamma_{i, k} \in \mathscr{S}_{n}} \int_{\Gamma_{i, k}}\left|1-\frac{\rho(z, \xi)^{2}}{\rho\left(z, \xi_{i, k}\right)^{2}}\right| \mathrm{d} \mu(\xi) .
\end{aligned}
$$

The same calculations that gave (8) show that

$$
\left|1-\frac{\rho(z, \xi)^{2}}{\rho\left(z, \xi_{i, k}\right)^{2}}\right| \leq 2\left(1-|z|^{2}\right)\left(\left|\frac{1-|\xi|^{2}}{|1-\bar{\xi} z|^{2}}-\frac{1-|\xi|^{2}}{\left|1-\bar{\xi}_{i, k} z\right|^{2}}\right|+\frac{\left.|| \xi_{i, k}\right|^{2}-|\xi|^{2} \mid}{\left|1-\bar{\xi}_{i, k} z\right|^{2}}\right) .
$$

For $\xi \in \Gamma_{i, k} \in \mathscr{Y}_{n}$, using $|1-\bar{\xi} z| \geq 2^{n-3}(1-|z|)$ we get

$$
\left(1-|\xi|^{2}\right)\left|\frac{1}{|1-\bar{\xi} z|^{2}}-\frac{1}{\left|1-\bar{\xi}_{i, k} z\right|^{2}}\right| \leq 2^{11} \frac{(1-|\xi|)\left|\xi-\xi_{i, k}\right|}{2^{3 n}(1-|z|)^{3}} \leq 2^{11} \frac{\left|\xi-\xi_{i, k}\right|}{2^{2 n}(1-|z|)^{2}}
$$


Similarly,

$$
\frac{\left.|| \xi_{i, k}\right|^{2}-|\xi|^{2} \mid}{\left|1-\bar{\xi}_{i, k} z\right|^{2}} \leq 2^{7} \frac{\left|\xi-\xi_{i, k}\right|}{2^{2 n}(1-|z|)^{2}}
$$

Adding up, we obtain

Hence

$$
\left|1-\frac{\rho(z, \xi)^{2}}{\rho\left(z, \xi_{i, k}\right)^{2}}\right| \leq 2^{14} \frac{\left|\xi-\xi_{i, k}\right|}{2^{2 n}(1-|z|)} .
$$

$$
E_{\mathscr{S}} \leq 2^{14} \sum_{n=N+1}^{\infty} \frac{1}{2^{2 n}(1-|z|)} \sum_{\Gamma_{i, k} \in \mathscr{Y}_{n}} \ell\left(\Gamma_{i, k}\right) \leq 2^{14}\left\|\mathrm{~d} s_{\mid \Gamma}\right\|_{C} \cdot 2^{-N} .
$$

Finally, we estimate the long $\operatorname{arcs} \Gamma_{i, k} \in \mathscr{L}_{n}$, for $n \geq N+1$. As the zeros on these arcs are well separated, one can expect only a small contribution from these arcs. We will use an auxiliary interpolating Blaschke product to find a bound for the $\mathscr{L}_{n}$-terms of (6). By the same reasoning that led to (8) and the triangle inequality,

$$
\begin{aligned}
E_{\mathscr{L}} & \stackrel{\text { def }}{=}\left|\sum_{n=N+1}^{\infty} \sum_{\Gamma_{i, k} \in \mathscr{L}_{n}} \int_{\Gamma_{i, k}} \log \frac{\rho(z, \xi)}{\rho\left(z, \xi_{i, k}\right)} \mathrm{d} \mu(\xi)\right| \\
& \leq 2 \sum_{n=N+1}^{\infty} \sum_{\Gamma_{i, k} \in \mathscr{L}_{n}} \int_{\Gamma_{i, k}}\left(1-|z|^{2}\right)\left(\frac{1-|\xi|^{2}}{|1-\bar{\xi} z|^{2}}+\frac{1-\left|\xi_{i, k}\right|^{2}}{\left|1-\bar{\xi}_{i, k} z\right|^{2}}\right) \mathrm{d} \mu(\xi) \\
& \leq 2^{2} \sum_{n=N+1}^{\infty} \sum_{\Gamma_{i, k} \in \mathscr{L}_{n}} \max _{\xi \in \bar{\Gamma}_{i, k}} \frac{\left(1-|z|^{2}\right)\left(1-|\xi|^{2}\right)}{|1-\bar{\xi} z|^{2}} .
\end{aligned}
$$

For each $\Gamma_{i, k} \in \mathscr{L}_{n}$, let $\zeta_{i, k} \in \Gamma_{i, k}$ be such that

$$
\frac{1-\left|\zeta_{i, k}\right|^{2}}{\left|1-\bar{\zeta}_{i, k} z\right|^{2}}=\max _{\xi \in \bar{\Gamma}_{i, k}} \frac{1-|\xi|^{2}}{|1-\bar{\xi} z|^{2}}
$$

and define $B_{\zeta}$ to be the Blaschke product with $\left\{\zeta_{i, k}\right\}$ as zeros. Now we reorder the summation, and sum with respect to the placement of the $\zeta_{i, k}$ instead. Then

$$
E_{\mathscr{L}} \leq 2^{3}(1-|z|) \sum_{n=0}^{\infty} \sum_{\zeta_{i, k} \in U_{n}} \frac{1-\left|\zeta_{i, k}\right|^{2}}{\left|1-\bar{\zeta}_{i, k} z\right|^{2}}
$$

where $U_{0}=Q_{z}$ and $U_{n}=2^{n} Q_{z} \backslash 2^{n-1} Q_{z}$ for $n \geq 1$. The scaling property (3) implies that at most four of the points $\zeta_{i, k}$ are contained in $2^{N-1} Q_{z}$. These must be close to the boundary, so

$$
2^{3}(1-|z|) \sum_{n=0}^{N-1} \sum_{\zeta_{i, k} \in U_{n}} \frac{1-\left|\zeta_{i, k}\right|^{2}}{\left|1-\bar{\zeta}_{i, k} z\right|^{2}} \leq 4 \cdot 2^{4} \cdot 2^{-2 N}
$$


For the rest of the terms, we then get

$$
2^{3}(1-|z|) \sum_{n=N}^{\infty} \sum_{\zeta_{i, k} \in U_{n}} \frac{1-\left|\zeta_{i, k}\right|^{2}}{\left|1-\bar{\zeta}_{i, k} z\right|^{2}} \leq 2^{8} \sum_{n=N}^{\infty} \frac{1}{2^{n}} \sum_{\zeta_{i, k} \in U_{n}} \frac{1-\left|\zeta_{i, k}\right|}{2^{n}(1-|z|)} \leq 2^{9} C_{\zeta} \cdot 2^{-N},
$$

where $C_{\zeta}$ is the Carleson norm of the measure $\sum\left(1-\left|\zeta_{i, k}\right|\right) \delta_{\zeta_{i, k}}$, which is bounded by a fixed multiple of $\left\|\mathrm{d} s_{\mid \Gamma}\right\|_{C}$. Thus $E_{\mathscr{L}} \leq 2^{9}\left(C_{\zeta}+1\right) \cdot 2^{-N}$.

We have now estimated the contribution from all the arcs $\Gamma_{i, k}$, and we have found that for some constant $C$,

$$
|\log | B_{1}(z)|-\log | I_{1}(z)|| \leq C \cdot 2^{-N} .
$$

This means that given $\varepsilon>0$, taking $N$ so that $C \cdot 2^{-N}<\varepsilon / 2$, we obtain

$$
|| B_{1}(z)|-| I_{1}(z)||<\varepsilon / 2,
$$

which is what we needed.

\section{Acknowledgments}

We are indebted to Arne Stray for his valuable comments on an earlier version of this paper. Part of this work was done while Hjelle was visiting the Universitat Autònoma de Barcelona and while Nicolau was visiting IMUB at the Universitat de Barcelona. It is a pleasure to thank both institutions for their support.

\section{References}

[Carleson 1958] L. Carleson, "An interpolation problem for bounded analytic functions", Amer. J. Math. 80 (1958), 921-930. MR 22 \#8129 Zbl 0085.06504

[Carleson 1962] L. Carleson, "Interpolations by bounded analytic functions and the corona problem”, Ann. of Math. (2) 76 (1962), 547-559. MR 25 \#5186 Zbl 0112.29702

[Frostman 1935] O. Frostman, "Potentiel d'équilibre et capacité des ensembles avec quelques applications á la théorie des fonctions", Medd. Lund. Univ. Math. Sem. 3 (1935). JFM 61.1262.02

[Garnett 1981] J. B. Garnett, Bounded analytic functions, Pure and Applied Mathematics 96, Academic Press, New York, 1981. MR 83g:30037 Zbl 0469.30024

[Garnett and Nicolau 1996] J. Garnett and A. Nicolau, "Interpolating Blaschke products generate $H^{\infty},$, Pacific J. Math. 173:2 (1996), 501-510. MR 97f:30050 Zbl 0871.30031

[Havin and Nikol'skiı̌ 1994] V. P. Havin and N. K. Nikol'skiı̌ (editors), Linear and complex analysis, problem book 3, part I, edited by V. P. Havin and N. K. Nikol'skǐ̌, Lecture Notes in Mathematics 1573, Springer, Berlin, 1994. MR 96c:00001a Zbl 0893.30037

[Jones 1981] P. W. Jones, "Ratios of interpolating Blaschke products", Pacific J. Math. 95:2 (1981), 311-321. MR 82m:30032 Zbl 0479.30021

[Lyubarskii and Malinnikova 2001] Y. Lyubarskii and E. Malinnikova, "On approximation of subharmonic functions", J. Anal. Math. 83 (2001), 121-149. MR 2002b:30043 Zbl 0981.31002

[Marshall 1976] D. E. Marshall, "Blaschke products generate $H^{\infty}$ ", Bull. Amer. Math. Soc. 82:3 (1976), 494-496. MR 53 \#5877 Zbl 0327.30029 
[Marshall and Stray 1996] D. E. Marshall and A. Stray, "Interpolating Blaschke products", Pacific J. Math. 173:2 (1996), 491-499. MR 97c:30042 Zbl 0855.30028

[Mortini and Nicolau 2004] R. Mortini and A. Nicolau, "Frostman shifts of inner functions", J. Anal. Math. 92 (2004), 285-326. MR 2005e:30088 Zbl 1064.30029

[Nicolau and Suárez $\geq 2006$ ] A. Nicolau and D. Suárez, "Approximation by invertible functions of $H^{\infty}$, Math. Scandinavica. To appear.

[Nikol'skiŭ 1986] N. K. Nikol'skiŭ, Treatise on the shift operator, Grundlehren der Mathematischen Wissenschaften 273, Springer, Berlin, 1986. MR 87i:47042 Zbl 0587.47036

[Seip 2004] K. Seip, Interpolation and sampling in spaces of analytic functions, University Lecture Series 33, American Math. Society, Providence, RI, 2004. MR 2005c:30038 Zbl 1057.30036

Received October 4, 2005.

GEIR ARne HJELle

DEPARTMENT OF MATHEMATICAL SCIENCES

Norwegian University of SCIENCE AND TECHNOLOGY

7491 TRONDHEIM

NORWAY

Current address:

Department of Mathematics

Washington University

St. Louis, MO 63130

United States

http://www.math.wustl.edu/ hjelle/

hjelle@math.wustl.edu

ARTUR NiCOLAU

Departament de Matemàtiques

UNIVERSITAT AUTÒNOMA DE BARCELONA

08193 BELLATERRA, BARCELONA

SPAIN

http://mat.uab.es/ artur/

artur@mat.uab.es 